Cita bibliográfica: Rabadán-Martín, I., Aguado-Correa, F. y Padilla-Garrido, N. (2021). ¿Son los establecimientos hoteleros y de restauración fieles a las certificaciones de los sistemas de gestión de calidad?. Investigaciones Turísticas (22), pp. 277-303. https://doi.org/10.14198/INTURI2021.22.12

\title{
¿Son los establecimientos hoteleros y de restauración fieles a las certificaciones de los sistemas de gestión de calidad?
}

\section{Do hospitality businesses retain and renew their quality management system certifications?}

Inmaculada Rabadán-Martín (iD, Universidad de Huelva, España rabadan@uhu.es

Francisco Aguado-Correa (iD, Universidad de Huelva, España agucor@uhu.es

Nuria Padilla-Garrido iD, Universidad de Huelva, España padilla@uhu.es

\section{RESUMEN}

La certificación de los sistemas de gestión de calidad ha tenido un fuerte crecimiento a lo largo de los años y ha venido demostrando su eficacia como elemento diferenciador al contribuir a mejorar la imagen de los establecimientos turísticos. Sin embargo, las certificaciones como la ISO 9001 o la "Q de calidad" han llegado o están llegando a un punto de saturación que parece indicar que no las hace atractivas como señales de calidad. Tomando como referencia todos los hoteles, apartamentos y servicios de restauración de Andalucía a los que se otorgó la certificación ISO 9001 o la "Q de calidad" entre 2006 y 2018, en este trabajo se analiza la evolución de estas dos certificaciones, incidiendo en la tasa de abandonos y en el grado de fidelidad a las mismas. Los resultados mostraron que los alojamientos tenían una mayor predisposición a elegir la ISO 9001, mientras que la restauración prefería la " $Q$ de calidad". Además, el análisis de la fidelidad nos mostró que, en ambos subsectores, el abandono de certificaciones es mucho mayor en el caso de la ISO 9001, incluso cuando se simultánea con la "Q de calidad", lo que es más habitual en los alojamientos. El análisis temporal individualizado de los establecimientos, reflejó que no ha habido un trasvase claro de certificaciones entre las dos normas. Como conclusión se plantea que los establecimientos de alojamiento y de restauración pueden estar manteniendo los procedimientos de los sistemas de gestión sin volverse a certificar.

Palabras clave: Hotelería y restauración; Certificación en calidad; ISO 9001; "Q de calidad"; Fidelidad. 


\section{ABSTRACT}

Quality management systems certification has grown strongly over the years and has proved its effectiveness as a differentiating element by supporting the improvement of the image of tourism establishments. However, certifications such as ISO 9001 or the 'Spanish Q for Quality in Tourism' have reached, or are reaching, a saturation point that seems to indicate they are no longer attractive as an indication of quality. Taking as a reference all the hospitality and restaurant services in Andalusia to which the ISO 9001 or the Q certifications were granted between 2006 and 2018, this paper analyses the evolution of these two certifications, in particular abandonment and retention rates. The results show that accommodation establishments were more inclined to choose ISO 9001, while the restaurant industry preferred the Q certification. Furthermore, the loyalty analysis reveals that, in both subsectors, abandonment of certification is much greater in the case of ISO 9001, even when combined with the $Q$ brand, as is more common in accommodation. The individualized temporal analysis of the establishments shows that there has been no clear transfer from one certification to the other. In conclusion, the findings suggest that hospitality and restaurant establishments may be willing to retain their quality management system certifications but are not pursuing recertification.

Keywords: Hospitality, Quality Certification, ISO 9001, Spanish Q for Quality in Tourism, Retention.

\section{INTRODUCCIÓN}

Durante décadas, y hasta la pandemia provocada por la COVID-19, el turismo ha venido experimentado un continuo crecimiento y una profunda diversificación que le han permitido situarse como una de las principales fuentes de ingresos de numerosos países.

España ha sido buena prueba de ello al convertirse en la economía más competitiva del mundo en términos turísticos y en el segundo destino más visitado del mundo (Calderwood et al., 2019). En concreto, 83,7 millones de turistas extranjeros visitaron España en 2019 (INE, 2020). En el caso de Andalucía, ámbito geográfico de este trabajo, lo hicieron 12,6 millones, a los que se añadieron 19,8 millones de turistas nacionales (IECA, 2020), situándola en cuarto lugar por afluencia turística de España, tras Cataluña, Islas Baleares y Canarias.

Entre los numerosos factores que han contribuido a alcanzar tales posiciones de privilegio destacan los recursos naturales y culturales, así como la calidad de la industria turística. A este respecto, numerosos establecimientos turísticos han emprendido el camino de la certificación en estándares reconocidos, buscando no sólo demostrar su compromiso con la calidad y la satisfacción del cliente, sino, también, exhibir una señal, en forma de certificado, sello o marca, que les permita comunicar las cualidades del producto o servicio para reducir la incertidumbre derivada de la asimetría de la información (Connelly et al., 2011; Terlaak y King, 2006), así como la diferenciación y prestigio frente al resto de competidores (Tarí et al., 2009; Casadesús et al., 2010; Rusjan y Alič, 2010; Ballina et al., 2019).

El etiquetado de productos y servicios o el uso de señales no es un aspecto nuevo. La teoría económica subyacente para el etiquetado de productos se remonta al trabajo de Stigler 
(1961) sobre la economía de la información, en el que la presentaba como un recurso valioso y, en particular, la información sobre los precios y la gran cantidad de tiempo necesario para recabarla. Aunque no la desarrollaba en su trabajo, también mencionaba la mayor dificultad de obtención de la información sobre la calidad de los productos y la dependencia del consumidor en la reputación de los mismos para ahorrar tiempo de búsqueda. Con posterioridad, Nelson (1970), siguiendo la misma línea, sostuvo que el problema para el consumidor a la hora de determinar los niveles de calidad era aún mayor que el de determinar los niveles de precio. En el mismo año, Akerlof (1970) señaló que, como la calidad puede no ser visible en el momento de la compra, existe un incentivo para que los vendedores prometan productos de alta calidad, pero comercialicen productos de baja calidad. Así pues, en algunos mercados, el consumidor se enfrenta a una mayor incertidumbre con respecto a la calidad que con respecto a los precios. Para reducir la incertidumbre del consumidor, Akerlof (1970) aludía a la emisión de señales como, por ejemplo, las garantías contractuales, la marca, la pertenencia a cadenas y las licencias o colegiaciones profesionales.

A lo largo del tiempo han ido apareciendo nuevas señales y la rentabilidad de su utilización, por parte de las empresas, ha dependido de la credibilidad de las mismas frente a los consumidores (Boulding y Kirmani, 1993).

En el ámbito turístico español se han venido empleando dos certificaciones de calidad que han actuado como señales: la certificación del sistema de gestión de la calidad basado en la norma ISO 9001 y la " $Q$ de calidad".

Por lo que respecta a la norma ISO 9001, elaborada por la Organización Internacional para la Estandarización (conocida como ISO, por sus siglas en inglés), tiene como objetivo certificar la gestión integral de la calidad de cualquier tipo de empresa, independientemente de su tamaño y sector. Si bien este certificado está fuertemente afianzado en los sectores industriales, también ha encontrado su hueco en el sector servicios. Los motivos para su implantación en el sector hotelero español son tanto internos como externos (Álvarez et al., 2012; AlonsoAlmeida et al., 2012; Tarí et al., 2014; Djofack y Robledo, 2017). Al respecto, Rubio-Andrada et al. (2011) destacan, principalmente, la mejora de los procesos internos y el trabajo de áreas funcionales, la presión de los competidores, la utilización de la calidad como parte de la estrategia empresarial y el mantenimiento de la posición competitiva en tiempos de crisis. Junto a los motivos, diversos estudios recogen, además, los beneficios que su implantación conlleva, entre los cuales destacan: el efecto positivo sobre la calidad del servicio, la lealtad y satisfacción del cliente, la relación con los proveedores y la participación en proyectos sociales (Rubio-Andrada et al., 2011), la competitividad y reputación online (Tarí et al., 2009; HerasSaizarbitoria et al., 2015), las mejoras en la organización empresarial, la imagen de marca o los resultados financieros (Djofack y Robledo, 2017; Tarí et al., 2009; Nicolau y Sellers, 2010; Rubio-Andrada et al., 2011; Ballina et al., 2019).

En lo referente a la " $Q$ de calidad", se trata de una certificación de calidad otorgada por el Instituto para la Calidad Turística Española (ICTE). Aunque es una norma nacional y, por tanto, no tiene el reconocimiento internacional de la ISO 9001, dispone, en cambio, de una serie de estándares adaptados a cada tipo de servicio turístico. Concretamente, la norma de calidad correspondiente al sector de Hoteles y Apartamentos Turísticos (UNE 182001) ha 
servido de inspiración para la creación de la primera norma mundial de calidad turística en hoteles, la ISO 22483, publicada en 2020, la cual recoge los requisitos mínimos de calidad que debe ofrecer un establecimiento hotelero, independientemente de su categoría y tipología, en cualquier parte del mundo.

La aplicación de la "Q de calidad" se ha asociado con la mejora de los resultados empresariales (Tarí-Guilló y Pereira-Moliner, 2012; Pereira-Moliner et al., 2016) y cuando se compara con otras certificaciones aporta numerosas ventajas (Tarí et al., 2009; Alonso-Almeida et al., 2013; Sánchez-Ollero et al., 2014; Ballina y Valdés, 2016) Ilegando, incluso, a aumentar la productividad del establecimiento en mayor medida que si éste hubiera implantado la ISO 9001 (Sánchez-Ollero et al., 2014). Además, existen estudios, como el de Ballina y Valdés (2016), que evidencian efectos sinérgicos cuando se combinan ambas certificaciones, concretamente, relacionados con la cuota de mercado y el precio medio por habitación.

A pesar de todas estas ventajas y del aumento del número de certificaciones ISO 9001 y " $Q$ de calidad" en el sector de hoteles y restauración en España, diversos estudios sugieren que su ritmo de crecimiento se ha ralentizado (Casadesús et al., 2010; Alonso-Almeida et al., 2013). Esta saturación ha ido acompañada, además, de un aumento de las tasas de abandono. En el caso de la ISO 9001, existen trabajos que han tratado de ahondar en las causas de esta falta de renovación destacando, principalmente, los costes asociados a la misma (Djofack y Robledo, 2017) así como el tiempo necesario para percibir los beneficios derivados de su implementación; tiempo que algunas empresas no están dispuestas a asumir (Nwankwo, 2000). Otros factores destacables que propician el abandono de la ISO 9001 son: (a) la falta de tiempo por parte de los empleados para participar activamente en el sistema implementado; (b) el temor de que el mantenimiento del certificado pueda implicar un aumento de los precios del servicio; (c) la consideración de que los beneficios derivados de la certificación pueden obtenerse, alternativamente, sin la implementación de un sistema de gestión de calidad; y (d) la elección de otros sistemas de gestión de la calidad, que puedan ser más eficientes y menos caros (Zimon y Dellana, 2019). Por lo que respecta a la "Q de calidad", y hasta donde sabemos, no tenemos constancia de que exista algún estudio que haya analizado las posibles causas de su falta de renovación.

Sin embargo, y a pesar de todos los trabajos que se han centrado en estudiar las causas de esta falta de renovación de la ISO 9001, ninguno de ellos ha analizado el nivel de fidelidad que tienen las empresas a este certificado ni a la " $Q$ de calidad". Tampoco han analizado si este nivel de fidelidad o la elección del certificado en sí dependen de la clase de establecimiento. Por tanto, y dado que no existe un claro conocimiento sobre estos aspectos, consideramos necesario profundizar en el tema. En este sentido, el objetivo de nuestro trabajo va a ser analizar el grado de fidelidad de los hoteles y servicios de restauración andaluces a las certificaciones ISO 9001 y "Q de calidad", estudiando si existen diferencias según el tipo de establecimiento. Además, analizaremos si existen establecimientos más propensos a elegir la ISO 9001, la " $Q$ de calidad" o ambas a la vez. Para ello, desarrollaremos, en primer lugar, el marco teórico en el que explicaremos la evolución de ambas certificaciones. Posteriormente expondremos la metodología utilizada, los resultados obtenidos, así como las principales conclusiones del estudio. 


\section{EVOLUCIÓN DE LAS CERTIFICACIONES ISO 9001 Y “Q DE CALIDAD” EN EL SECTOR DE HOTELES Y RESTAURACIÓN}

\subsection{Evolución mundial de ISO 9001}

La norma ISO 9001 es la herramienta de gestión de la calidad más extendida en el mundo. Según el informe ISO Survey 2018 (ISO, 2019), esta certificación ha mostrado una evolución creciente hasta el año 2010, momento a partir del cual se estabiliza en torno al millón de certificados, tal y como puede comprobarse en el Gráfico 1.

Por lo que respecta al sector turístico, hubiera sido razonable suponer que la ISO 9001 también hubiera tenido una amplia aceptación en el mismo. Sin embargo, parece que este no ha sido el caso. Según el ISO Survey 2018 (ISO, 2019), de los 872.702 certificados en todo el mundo, sólo 4.429 correspondían a hoteles y restauración, es decir el 0,51\%. Como se aprecia en el Gráfico 2, tras una tendencia inicial creciente, a partir de 2007 el número de certificaciones ISO 9001 en este sector tiene un comportamiento inestable, con un claro retroceso en 2017. Si se analiza en términos porcentuales respecto al total de certificaciones, el sector de hoteles y restauración alcanzó un valor mínimo en 2000 y un valor máximo en 1999, aunque en la mayoría de los años osciló entre el $0,5 \%$ y el $0,65 \%$.

Gráfico 1. Evolución global del número de certificaciones ISO 9001 en el ámbito mundial $(1993-2017)^{1}$

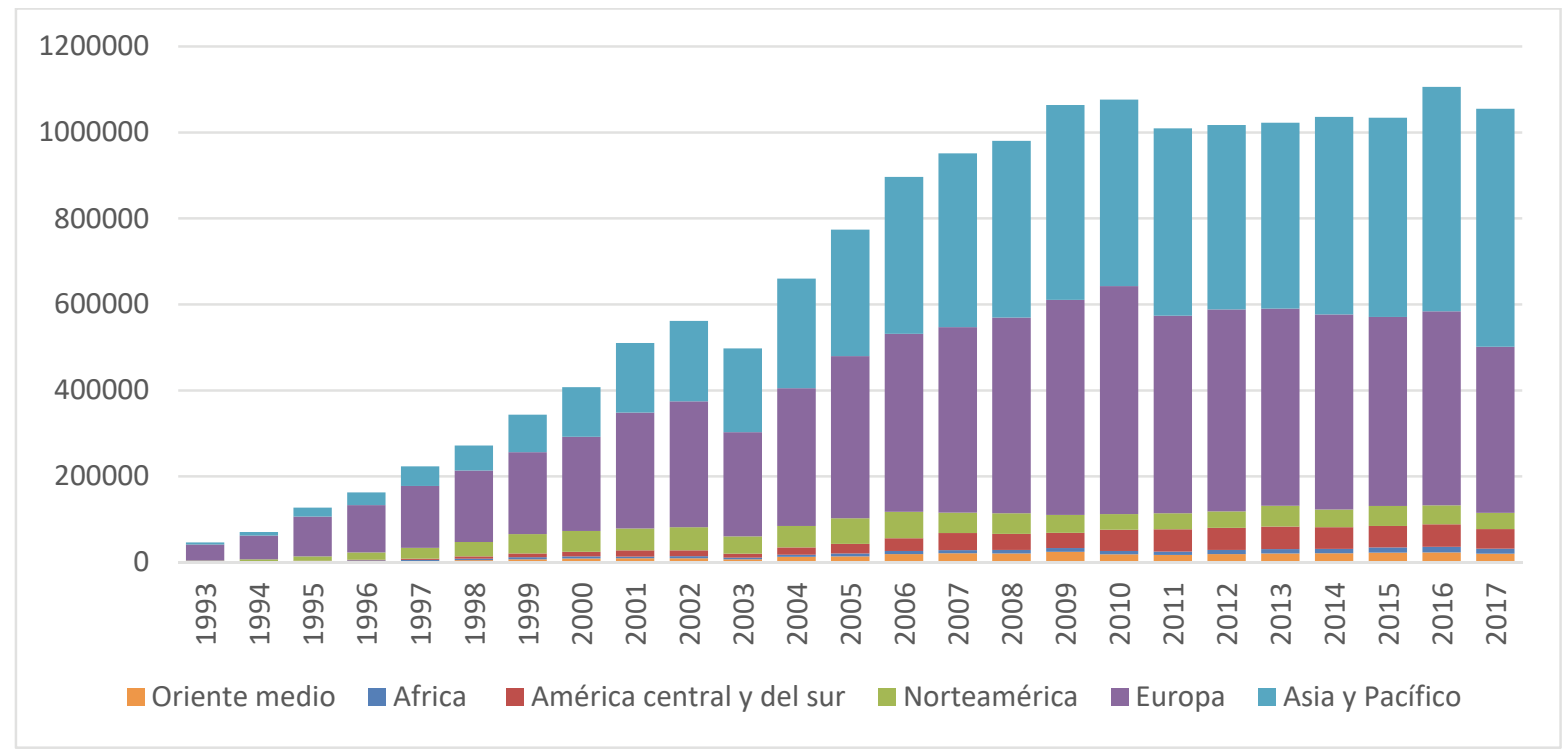

Fuente: elaborado a partir de ISO (2019).

1. El informe ISO Survey 2018 (ISO, 2019) ofrece los datos de certificaciones desde 1993 a 2017 por áreas geográficas, no disponiéndose de datos relativos a 2018. Según este informe, la reducción en el número de certificados globales de 2017 con respecto a 2016 se debe, en parte, a cambios en la forma en que recibieron los datos. 
Gráfico 2. Evolución absoluta y relativa de las certificaciones ISO 9001 en el sector de hoteles y restauración a nivel mundial (1998-2017)²

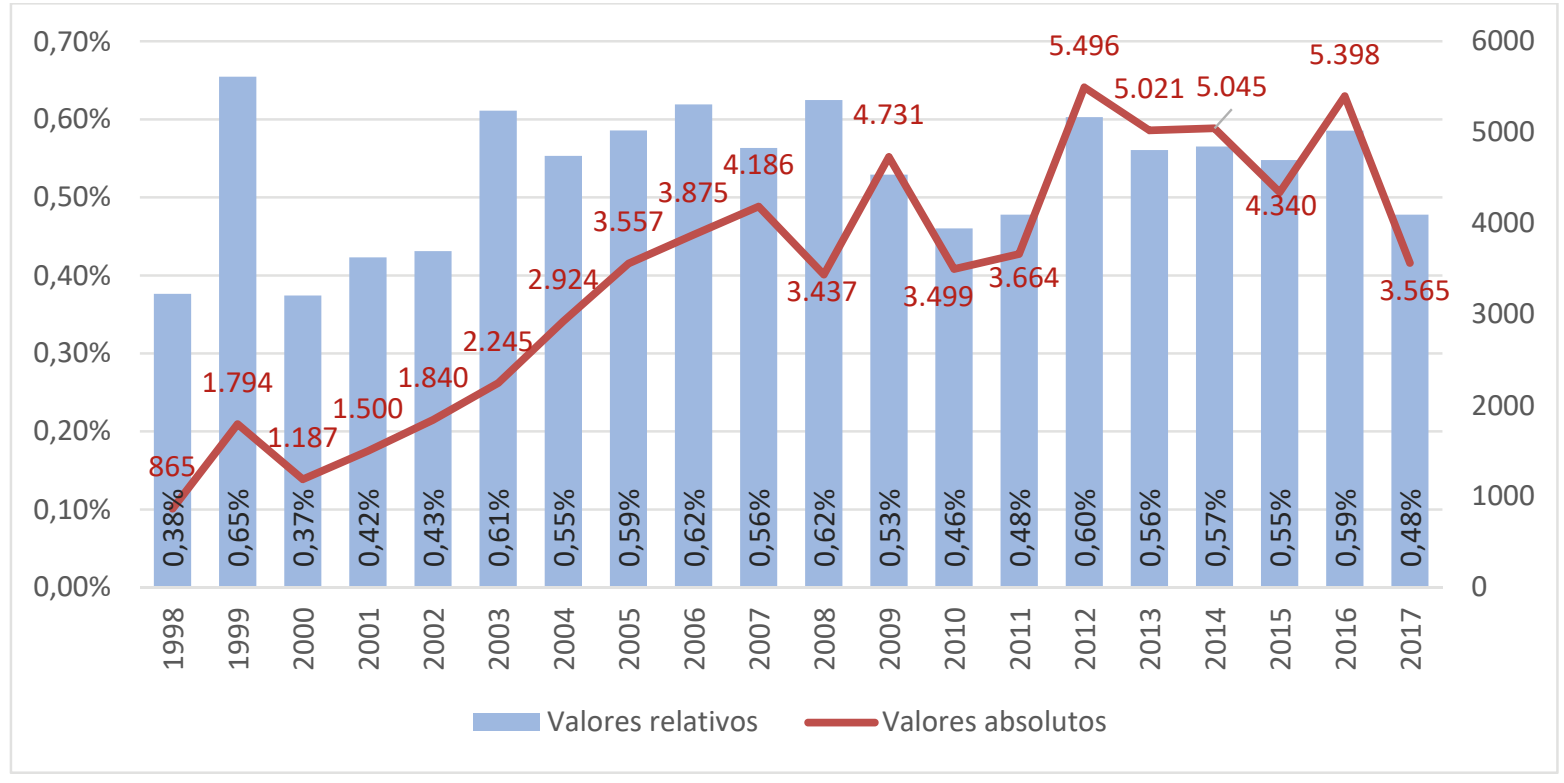

Fuente: elaborado a partir de ISO (2019).

\subsection{Evolución en España y Andalucía de ISO 9001 y "Q de calidad"}

La baja penetración de la ISO 9001 en el sector turístico contrasta, en cambio, con la elevada implantación de la " $Q$ de calidad". En la Tabla 1, en la que se muestra la evolución de ambos certificados en España y Andalucía, podemos observar, además del claro predominio de la "Q de calidad" frente a la ISO 9001, una mayor disminución en el número de certificados de la ISO 9001 (54\%), en España, con respecto a la "Q de calidad" (20\%), si se compara 2009 frente a 2018.

2. El informe ISO (2019) solo ofrece datos mundiales por sectores para el periodo 1998 a 2017. 
Tabla 1. Evolución del número de certificaciones ISO 9001 y " $Q$ de calidad" en el sector de hoteles y restauración de España y Andalucía (2009-2018) ${ }^{3,4}$

\begin{tabular}{|r|r|r|r|r|r|r|}
\hline & \multicolumn{3}{|c|}{ ISO 9001 } & \multicolumn{3}{c|}{ Q de calidad } \\
\hline \multicolumn{1}{|c|}{ Año } & España & Andalucía & $\begin{array}{c}\text { \% ISO Andalucía } \\
\text { vs. España }\end{array}$ & España & Andalucía & $\begin{array}{c}\text { \% Q Andalucía } \\
\text { vs. España }\end{array}$ \\
\hline $\mathbf{2 0 0 9}$ & 524 & 139 & $26,5 \%$ & 731 & 118 & $16,1 \%$ \\
\hline $\mathbf{2 0 1 0}$ & 517 & 151 & $29,2 \%$ & 1190 & 124 & $10,4 \%$ \\
\hline $\mathbf{2 0 1 1}$ & 469 & 123 & $26,2 \%$ & 1194 & 189 & $15,8 \%$ \\
\hline $\mathbf{2 0 1 2}$ & 530 & 79 & $14,9 \%$ & 1120 & 189 & $16,9 \%$ \\
\hline $\mathbf{2 0 1 3}$ & 373 & 91 & $24,4 \%$ & 1089 & 183 & $16,8 \%$ \\
\hline $\mathbf{2 0 1 4}$ & 287 & 94 & $32,8 \%$ & 1062 & 173 & $16,3 \%$ \\
\hline $\mathbf{2 0 1 5}$ & - & 110 & - & 1075 & 172 & $16,0 \%$ \\
\hline $\mathbf{2 0 1 6}$ & - & 42 & - & 1165 & 193 & $16,6 \%$ \\
\hline $\mathbf{2 0 1 7}$ & 207 & 45 & $21,7 \%$ & 674 & 111 & $16,5 \%$ \\
\hline $\mathbf{2 0 1 8}$ & 238 & 26 & $10,9 \%$ & 589 & 126 & $21,4 \%$ \\
\hline
\end{tabular}

Fuente: elaborado a partir de ISO (2019), ICTE (2019), Junta de Andalucía (2010 a 2019).

Estas cifras están en consonancia con las reflejadas en otros estudios (Casadesús et al., 2010; Alonso-Almeida et al., 2013; Djofack y Robledo, 2017), en los cuales se concluye que la ISO 9001 ha alcanzado su nivel de saturación y declive en el sector de hoteles y restauración en España, mientras que Alonso-Almeida et al. (2013) pronosticaban la saturación de la " $Q$ de calidad" en $2019^{5}$.

Por lo que respecta a Andalucía, en la Tabla 1 puede observarse un claro predominio de la "Q de calidad" frente a la ISO 9001 en cada uno de los años estudiados. Si analizamos, en cambio, la evolución individual de la " $Q$ de calidad" en el periodo de estudio, el número de certificaciones activas aumentó, aproximadamente, un 7\% (pasando de 118 a 126) frente a una caída drástica de la ISO 9001 (81\%). Ahora bien, teniendo en cuenta la proporción de certificaciones que aporta Andalucía al total de España, la primera aumentó casi un 33\% y la segunda disminuyó casi un 59\%.

3. Es necesario aclarar que solo contamos con datos disponibles a partir de 2009 y que: a) en el año 2015, el valor de la ISO 9001 nacional se desechó debido a errores propios del informe de resultados de la "Encuesta de certificaciones ISO 9001"; y b) que no se disponen de datos con respecto al número de certificaciones en 2016 debido a que ISO no ha publicado el archivo "Data per sector and per country" relativo a dicho año.

4. Las categorías o códigos considerados en el cómputo de certificados de la " $Q$ de calidad" en este estudio son: Hoteles y apartamentos turísticos; y servicios de restauración.

5. Para realizar el pronóstico consideraron los siguientes códigos: Alojamientos rurales; Alojamientos sostenibles; Balnearios; Campings; Hoteles y apartamentos turísticos; y Servicios de restauración. 


\subsection{Evolución por tipo de establecimiento en Andalucía}

Si se realiza un análisis por tipo de establecimiento (Gráfico 4), podemos observar que, en Andalucía, los servicios de catering han optado, por la ISO 9001 mientras que los restaurantes, bares y cafeterías se han decantado, mayoritariamente, por la " $Q$ de calidad".

Gráfico 4. Evolución del número de certificaciones ISO 9001 y "Q de calidad”, según el tipo de establecimiento, en el sector de hoteles y restauración de Andalucía (2006-2018)

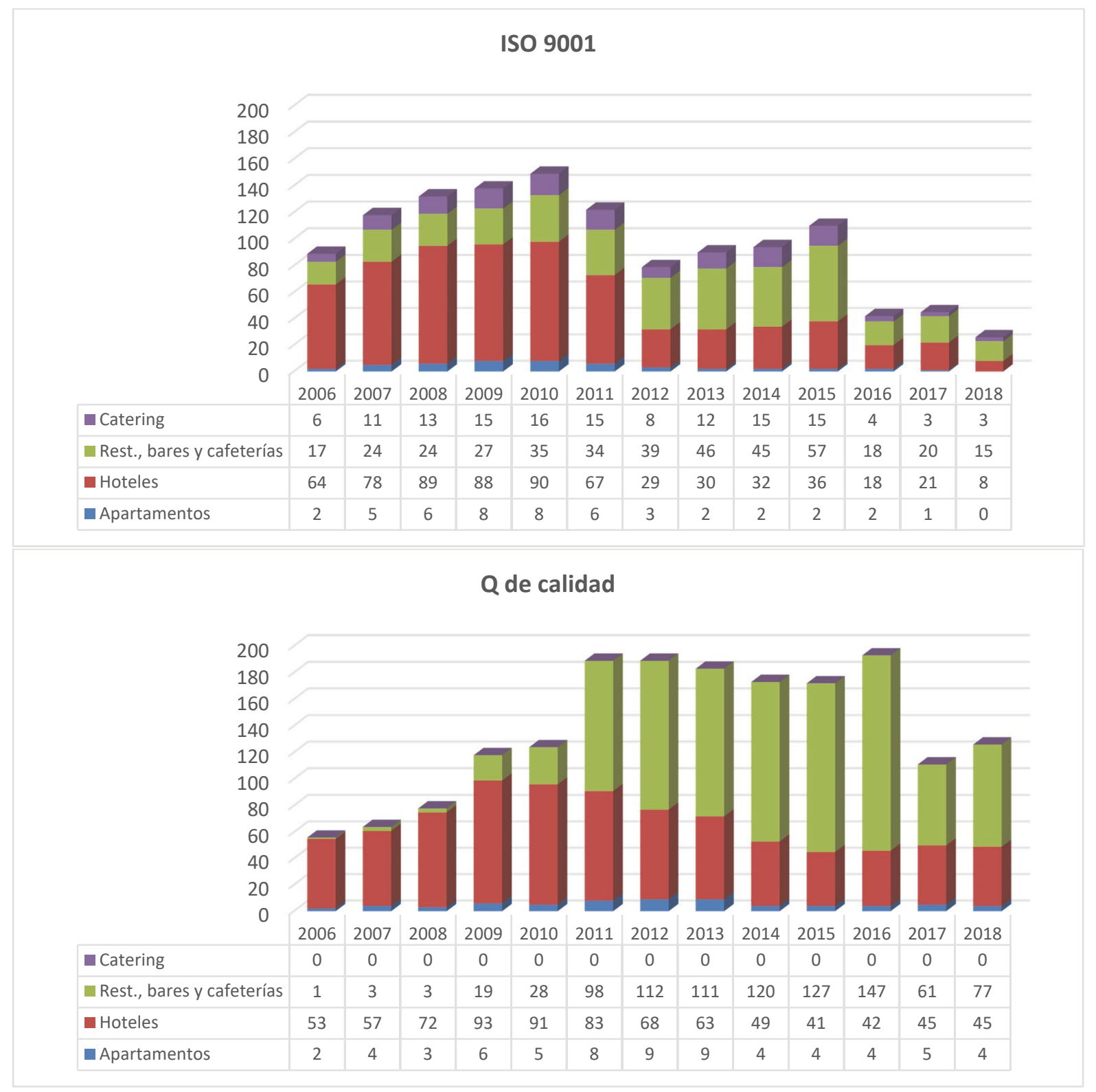

Fuente: elaborado a partir de Junta de Andalucía (2007 a 2019).

Teniendo en cuenta las cifras anteriores, podemos concluir que, tanto en España como en Andalucía, se ha producido un claro descenso en el número de certificados ISO 9001 en el 
sector de hoteles y restauración, mientras que la " $Q$ de calidad" ha tenido una fuerte caída a partir de 2016. Sin embargo, este análisis preliminar no nos permite conocer, año a año, si a pesar de incorporarse nuevas empresas al sistema, éstas superan en número a las que lo abandonan. Además, tampoco conocemos el grado de mantenimiento de los certificados una vez conseguidos.

En los siguientes apartados trataremos de dar respuesta a estas cuestiones.

- ¿Hay diferencias significativas en la elección de certificaciones ISO 9001 y " $Q$ de calidad" según el subsector?

- ¿Hay tipos de establecimientos más propensos a utilizar las dos certificaciones?

- ¿Existen diferencias de fidelidad entre tipos de establecimientos?

\section{MARCO TEÓRICO Y PLANTEAMIENTO DE HIPÓTESIS}

\subsection{Certificaciones ISO 9001 y "Q de calidad" en el sector alojamiento y restauración español}

La importancia de España como destino turístico ha propiciado los estudios de la calidad en el alojamiento y restauración, donde se incluyen las certificaciones de calidad ISO 9001 y " $Q$ de calidad", analizando las motivaciones de los establecimientos para certificarse, los efectos internos y externos que pueden tener sobre las empresas certificadas, así como sus niveles de difusión y tendencia (Tabla 2).

Tabla 2. Principales estudios de la aplicación de las certificaciones de ISO 9001 y " $Q$ de calidad" en el sector de alojamiento y restauración español

\begin{tabular}{|c|c|c|c|}
\hline Autor/es & Certificados & Temática & Principales resultados \\
\hline $\begin{array}{l}\text { Ballina, Valdés y Del } \\
\text { Valle (2019) }\end{array}$ & $\begin{array}{l}\text { ISO } 9001, \text { "Q } \\
\text { de calidad" y } \\
\text { otros certifica- } \\
\text { dos }\end{array}$ & Efectos & $\begin{array}{l}\text { Las certificaciones de calidad mejoran la rentabi- } \\
\text { lidad por habitación y el beneficio global de los } \\
\text { hoteles }\end{array}$ \\
\hline $\begin{array}{l}\text { Iglesias, Ballina y Caso } \\
\text { (2018) }\end{array}$ & "Q de calidad" & $\begin{array}{l}\text { Motivaciones } \\
\text { / Satisfacción }\end{array}$ & $\begin{array}{l}\text { Diferencias en el comportamiento de las cadenas } \\
\text { hoteleras según su tamaño, segmento de mercado, } \\
\text { origen de los clientes y concentración geográfica de } \\
\text { sus establecimientos }\end{array}$ \\
\hline $\begin{array}{l}\text { Álvarez-García, Del } \\
\text { Río-Rama y Simonetti } \\
(2017)\end{array}$ & "Q de calidad" & Efectos & $\begin{array}{l}\text { Relación positiva entre los procesos de mejora } \\
\text { continua y la política/planificación de la calidad y la } \\
\text { gestión de los procesos }\end{array}$ \\
\hline $\begin{array}{l}\text { Djofack y Robledo } \\
\text { (2017) }\end{array}$ & ISO 9001 & $\begin{array}{l}\text { Motivaciones } \\
\text { / Satisfacción }\end{array}$ & $\begin{array}{l}\text { Razones internas, más que externas, en la decisión } \\
\text { de implementación de ISO } 9001 \text {. Tamaño, edad y } \\
\text { consultoría afectan al coste y tiempo para la imple- } \\
\text { mentación. Satisfacción con ISO } 9001\end{array}$ \\
\hline $\begin{array}{l}\text { Ballina y Valdés } \\
\text { (2016) }\end{array}$ & $\begin{array}{l}\text { ISO } 9001, \text { "Q } \\
\text { de calidad" y } \\
\text { certificados } \\
\text { regionales }\end{array}$ & Efectos & $\begin{array}{l}\text { ISO } 9001 \text { mejora principalmente los resultados } \\
\text { financieros, mientras que la " } Q \text { de Calidad" los } \\
\text { comerciales. La combinación de "Q de Calidad" con } \\
\text { otras normas mejora el rendimiento económico }\end{array}$ \\
\hline
\end{tabular}




\begin{tabular}{|c|c|c|c|}
\hline Autor/es & Certificados & Temática & Principales resultados \\
\hline $\begin{array}{l}\text { Fuentes, Hernández y } \\
\text { Morini (2016) }\end{array}$ & "Q de calidad" & Efectos & $\begin{array}{l}\text { El efecto de la mejora de la calidad percibida en los } \\
\text { hoteles con "Q de calidad" disminuye al aumentar } \\
\text { el número de estrellas. El efecto positivo de la certi- } \\
\text { ficación no se observa en todas las CC. AA. }\end{array}$ \\
\hline $\begin{array}{l}\text { Pereira-Moliner, } \\
\text { Tarí, Molina-Azorín y } \\
\text { López-Gamero (2016) }\end{array}$ & "Q de calidad" & Efectos & $\begin{array}{l}\text { La interiorización de los requisitos de la marca “Q } \\
\text { de calidad" tiene beneficios para los clientes, em- } \\
\text { pleados, sociedad y organización }\end{array}$ \\
\hline $\begin{array}{l}\text { Heras-Saizarbitoria, } \\
\text { Arana y Borial (2015) }\end{array}$ & ISO 9001 & Efectos & $\begin{array}{l}\text { Los hoteles más grandes y con más estrellas son } \\
\text { más propensos a tener certificación ISO 9001, si } \\
\text { bien no obtienen mejor satisfacción de los clientes }\end{array}$ \\
\hline $\begin{array}{l}\text { Sánchez-Ollero, Gar- } \\
\text { cía-Pozo y Marchan- } \\
\text { te-Mera (2014) }\end{array}$ & $\begin{array}{l}\text { ISO } 9001, \mathrm{Q} \text { de } \\
\text { calidad y otras } \\
\text { certificaciones } \\
\text { internas }\end{array}$ & Efectos & $\begin{array}{l}\text { Los hoteles con ISO } 9001 \text { obtienen menos produc- } \\
\text { tividad. Los que ofrecen "Q de Calidad" consiguen } \\
\text { una productividad mayor, a igualdad de carac- } \\
\text { terísticas con los no certificados }\end{array}$ \\
\hline $\begin{array}{l}\text { Tarí, Heras-Saizarbito- } \\
\text { ria y Dick (2014) }\end{array}$ & "Q de calidad" & $\begin{array}{l}\text { Motivaciones } \\
\text { / Efectos }\end{array}$ & $\begin{array}{l}\text { Los hoteles que buscan la certificación por razones } \\
\text { internas desarrollan herramientas de mejor calidad } \\
\text { y tienen mayores niveles de beneficio }\end{array}$ \\
\hline $\begin{array}{l}\text { Alonso-Almeida, } \\
\text { Marimon y Bernardo } \\
(2013)\end{array}$ & $\begin{array}{l}\text { ISO } 9001 \text { y "Q } \\
\text { de calidad" }\end{array}$ & $\begin{array}{l}\text { Difusión / } \\
\text { tendencia }\end{array}$ & $\begin{array}{l}\text { Mientras que la "Q de Calidad" estaría en fase de } \\
\text { crecimiento, la ISO } 9001 \text { parece haber alcanzado su } \\
\text { punto de saturación }\end{array}$ \\
\hline $\begin{array}{l}\text { Álvarez, Fraiz y del Río } \\
\text { (2013) }\end{array}$ & "Q de calidad" & $\begin{array}{l}\text { Barreras de } \\
\text { entrada }\end{array}$ & $\begin{array}{l}\text { Las barreras de entrada más importantes son: falta } \\
\text { de tiempo para tareas de calidad, resistencia al } \\
\text { cambio y a asumir nuevas responsabilidades por } \\
\text { parte de los empleados }\end{array}$ \\
\hline $\begin{array}{l}\text { Pertusa, Tarí, Perei- } \\
\text { ra, Molina y López } \\
(2013)\end{array}$ & $\begin{array}{l}\text { ISO 9001, “Q } \\
\text { de calidad” y } \\
\text { EFQM }\end{array}$ & Efectos & $\begin{array}{l}\text { Los hoteles certificados desarrollan mayores prácti- } \\
\text { cas de gestión de la calidad, resultados empresari- } \\
\text { ales, formalización y coordinación }\end{array}$ \\
\hline $\begin{array}{l}\text { Alonso-Almeida, } \\
\text { Rodríguez-Antón y } \\
\text { Rubio-Andrada (2012) }\end{array}$ & $\begin{array}{l}\text { ISO } 9000 \text { y "Q } \\
\text { de calidad" }\end{array}$ & $\begin{array}{l}\text { Motivaciones } \\
\text { / Efectos }\end{array}$ & $\begin{array}{l}\text { Cuando la certificación está impulsada por factores } \\
\text { externos mejora la imagen para los stakeholders y } \\
\text { se obtienen mayores beneficios. Si los motivos son } \\
\text { internos mejoran las operaciones y servicios y au- } \\
\text { menta la satisfacción de los empleados }\end{array}$ \\
\hline $\begin{array}{l}\text { Álvarez, Fraiz y del Río } \\
(2012)\end{array}$ & "Q de calidad" & Motivaciones & $\begin{array}{l}\text { Importancia de las razones internas sobre el resto } \\
\text { (externas de mercado o externas de exigencias) }\end{array}$ \\
\hline $\begin{array}{l}\text { Tarí-Guilló y Perei- } \\
\text { ra-Moliner (2012) }\end{array}$ & "Q de calidad" & Efectos & $\begin{array}{l}\text { La certificación tiene efectos positivos en los re- } \\
\text { sultados y el tamaño de la cadena no es un factor } \\
\text { importante para certificarse }\end{array}$ \\
\hline $\begin{array}{l}\text { Alonso-Almeida y } \\
\text { Rodríguez-Antón } \\
\text { (2011) }\end{array}$ & $\begin{array}{l}\text { ISO } 9001, \text { "Q } \\
\text { de calidad", ISO } \\
\text { 14001, EMAS, } \\
\text { OHSAS } 18000 \\
\text { y otros certifi- } \\
\text { cados }\end{array}$ & Efectos & $\begin{array}{l}\text { La adopción de normas de gestión es ventajosa } \\
\text { para los hoteles independientes, que son los esta- } \\
\text { blecimientos que más aplican sistemas de gestión } \\
\text { normalizados }\end{array}$ \\
\hline $\begin{array}{l}\text { Rubio-Andrada, } \\
\text { Alonso-Almeida y } \\
\text { Rodríguez-Antón } \\
\text { (2011) }\end{array}$ & $\begin{array}{l}\text { ISO } 9001 \text { y "Q } \\
\text { de calidad" }\end{array}$ & $\begin{array}{l}\text { Motivaciones } \\
\text { / Efectos }\end{array}$ & $\begin{array}{l}\text { Los factores de motivación son: reacción al merca- } \\
\text { do, eficiencia e imagen. Entre los factores de im- } \\
\text { pacto destacan: clientes y operaciones comerciales, } \\
\text { rendimiento financiero e imagen pública }\end{array}$ \\
\hline
\end{tabular}




\begin{tabular}{|l|l|l|l|}
\hline \multicolumn{1}{|c|}{ Autor/es } & \multicolumn{1}{c|}{ Certificados } & \multicolumn{1}{c|}{ Temática } & \multicolumn{1}{c|}{ Principales resultados } \\
\hline $\begin{array}{l}\text { Casadesús, Marimon } \\
\text { y Alonso (2010) }\end{array}$ & $\begin{array}{l}\text { ISO 9001, “Q } \\
\text { de calidad” y } \\
\text { ISO 14001 }\end{array}$ & $\begin{array}{l}\text { Difusión / } \\
\text { tendencia }\end{array}$ & $\begin{array}{l}\text { Previsión de aumento de la estandarización de la } \\
\text { gestión de la calidad en el turismo }\end{array}$ \\
\hline $\begin{array}{l}\text { Tarí, Claver-Cortés, } \\
\text { Pereira-Moliner, Moli- } \\
\text { na-Azorín (2009) }\end{array}$ & $\begin{array}{l}\text { ISO 9001 y “Q } \\
\text { de calidad” }\end{array}$ & Efectos & $\begin{array}{l}\text { Niveles de rendimiento más altos para todas las } \\
\text { variables. Los hoteles certificados son significati- } \\
\text { vamente más grandes, ofrecen más instalaciones, } \\
\text { están afiliados a cadenas, están más comprometi- } \\
\text { dos con la gestión de la calidad y ofrecen más ca- } \\
\text { pacitación a sus empleados }\end{array}$ \\
\hline $\begin{array}{l}\text { Claver, Tarí y Pereira } \\
\text { (2006) }\end{array}$ & $\begin{array}{l}\text { ISO 9001 y “Q } \\
\text { de calidad” }\end{array}$ & $\begin{array}{l}\text { Motivaciones } \\
\text { / Efectos }\end{array}$ & $\begin{array}{l}\text { Las razones más importantes para adoptar y cer- } \\
\text { tificar los sistemas de calidad son internas y el im- } \\
\text { pacto en el rendimiento financiero es bajo }\end{array}$ \\
\hline
\end{tabular}

Elaboración propia.

La elección de la ISO 9001, de la "Q de calidad", o de ambas a la vez, puede venir determinada por las motivaciones y beneficios derivados de su implantación, así como por las diferencias existentes entre los alojamientos y los servicios de restauración. Si bien el análisis de estas certificaciones en España ha estado más centrado en los alojamientos, algunos estudios también incluyen a las empresas de restauración, ofreciendo resultados globales (Djofack y Robledo, 2017; Casadesús et al., 2010) o diferenciados para cada subsector (Pereira-Moliner et al., 2016; Alonso-Almeida et al., 2013). En este último caso, de acuerdo con Alonso-Almeida et al. (2013), los restaurantes podrían inclinarse más por la certificación " $Q$ de calidad", dado que tiene una versión específica para este tipo de establecimientos y permitiría implementar una norma de calidad con menores costes que la ISO 9001. Sin embargo, si bien los niveles de interiorización de la " $Q$ de calidad" en los restaurantes pueden ser similares a los de los hoteles, los beneficios obtenidos son ligeramente inferiores, tanto en relación con los clientes como con empleados, sociedad y organización (Pereira-Moliner et al., 2016). Profundizando en las diferencias entre el alojamiento y la restauración nos planteamos las siguientes hipótesis:

H1a: En los alojamientos hay diferencias significativas entre la proporción de certificación de ISO 9001 y Q de calidad.

H1b: En la restauración hay diferencias significativas entre la proporción de certificación de ISO 9001 y Q de calidad.

H1c: La elección conjunta de ISO 9001 y "Q de calidad" está relacionada con el tipo de establecimiento

\subsection{Evolución, fidelidad y abandono de certificaciones ISO 9001 y “Q de calidad”}

El estudio de la evolución del número de certificados de calidad ISO 9001, así como su previsión futura, ha sido ampliamente analizada de manera global en los últimos años (Ikram et al., 2020; Mastrogiacomo et al., 2020; Rodríguez-Arnaldo y Martínez-Lorente, 2020; Franceschini et al., 2010; Marimon et al., 2010; Sampaio et al., 2011; Cabecinhas et al., 2018), llegando a la conclusión que la difusión de las certificaciones ISO 9001 puede descomponerse 
en cuatro fases consecutivas: (1) crecimiento, en la que el número de certificaciones crece de manera constante; (2) madurez, o saturación, en la que el volumen de certificaciones se estabiliza; (3) declive, en la que disminuye el número de certificados; y (4) post-declive, en la que el total de certificaciones se estabiliza en un determinado nivel (Mastrogiacomo et al., 2020). La tendencia general parece ser la misma en los distintos países, pero la temporalidad y la evolución varían de país a país. En el caso del sector de alojamiento y restauración en España, la certificación ISO 9001 parece haber alcanzado su punto de saturación (Casadesús, 2010; Alonso-Almeida et al., 2013).

Los motivos de que la ISO 9001 haya dejado atrás la fase de crecimiento puede estar en el abandono de las certificaciones, pero también en las barreras de entrada o en la reducción del atractivo para las restantes empresas no certificadas (Zimon y Dellana, 2019; Simon y Kafel, 2018; Cândido et al., 2019; Kafel y Simon, 2017; Sansalvador y Brotins, 2015). Profundizando en ese ámbito, otros trabajos analizan el impacto que tienen sobre las empresas la cancelación de esta certificación (Alič, 2014; Cândido et al. 2016).

En relación con la evolución de la " $Q$ de calidad", y como ya hemos mencionado, hay trabajos que mostraban que se encontraba en la fase de crecimiento (Casadesús, 2010; Alonso-Almeida et al., 2013) y pronosticaban que no llegaría a la saturación hasta 2019 (AlonsoAlmeida et al., 2013).

Sin embargo, el estudio de la tendencia a abandonar las certificaciones ISO 9001 y " $Q$ de calidad" en el sector turístico apenas ha sido abordado. A este respecto, podríamos asociar una mayor satisfacción con las certificaciones con una menor tendencia a abandono, o mayor fidelidad a la norma. En este sentido, el trabajo de Djofack y Robledo (2017) concluía que los hoteles que tenían implantado el certificado ISO 9001 estaban generalmente satisfechos con la certificación. Por otro lado, el trabajo de Iglesias et al. (2018) analizaba la tendencia de incrementar o reducir la proporción de hoteles certificados con la " $Q$ de calidad", en el caso de cadenas hoteleras en España, llegando a la conclusión de que las cadenas más pequeñas, con mayor dispersión geográfica, o alta proporción de turistas extranjeros tenían una mayor tendencia a salir del sistema de certificación. Ballina et al. (2019) utilizaban como variable principal los cambios en la certificación para analizar los efectos que tenían en los resultados el hecho de incorporarse o abandonar los certificados ISO 9001 o "Q de calidad", si bien centraban su estudio en las nuevas incorporaciones a la certificación, concluyendo que mejoraban significativamente.

La literatura, por tanto, se ha centrado en los efectos o las causas de las descertificaciones, sin embargo, no se ha hecho un estudio detallado de los datos relativos al abandono o la fidelidad por subsectores. Si bien, el estudio de Alonso-Almeida et al. (2013) incluye casos tanto de hoteles como de restaurantes, las diferencias en la propensión a mantener los certificados ISO 9001 o "Q de calidad" según el subsector, no han sido analizadas. Considerando, por tanto, que el cumplimiento de los efectos esperados asociados a una certificación puede influir en su nivel de fidelidad y ésta, a su vez, puede ser diferente en función de si se trata de un alojamiento o un servicio de restauración, nos plantemos las siguientes hipótesis: 
H2a: El nivel de fidelidad de los alojamientos a la ISO 9001 es diferente al alcanzado con la " $Q$ de calidad"

H2b: El nivel de fidelidad de la restauración a la ISO 9001 es diferente al alcanzado con la " $Q$ de calidad"

\section{METOdOLOGÍA}

Para alcanzar nuestro objetivo, se analizaron los hoteles y servicios de restauración de Andalucía a los que se otorgó alguna de las dos certificaciones entre 2006 y 2018. Para ello se consultó el directorio de establecimientos y espacios certificados, publicado anualmente por la Junta de Andalucía (2007-2019). Tras analizar los apartados de los establecimientos hoteleros y apartamentos turísticos, bares y cafeterías, y otros servicios de restauración, 603 empresas proporcionaron datos analizables. Esto nos permitió, en primer lugar, conocer cuántos establecimientos habían obtenido, en alguno de los años, la "Q de calidad", la ISO 9001, o ambas a la vez.

Tras ello, para analizar la evolución de estas certificaciones, se comenzó realizando un estudio individualizado de cada empresa y certificado, anotando en qué momentos disponían de cada uno de ellos. De este modo, en cada año del estudio, los establecimientos se clasificaron en tres grupos: (a) empresas entrantes o que obtenían el certificado ese año; (b) empresas que mantenían activo el mismo certificado que el año anterior; y (c) empresas salientes o que no renovaban el certificado.

Con el propósito de distinguir, en este último caso, si la empresa había abandonado voluntariamente el sello o era a causa del cierre del establecimiento, en enero de 2019 se hizo una búsqueda de sus páginas web o, en el caso de no tenerlas, de los horarios de apertura (más común en el análisis de los bares y restaurantes). En el caso de que el establecimiento estuviese cerrado temporalmente, debido a las características de su demanda, lo que es habitual en los chiringuitos de playa, se analizó la fecha del último comentario en TripAdvisor. Una vez distinguidas las empresas que podrían estar cerradas, se utilizó la base de datos SABI (Sistema de Análisis de Balances Ibéricos) para determinar la fecha de la última presentación de las cuentas anuales, y se comprobó si coincidía con el último año en el que dispusieron de certificado.

Posteriormente, se estudió el grado de fidelidad a los certificados una vez conseguidos. Si la empresa se certificaba por primera vez en 2018, último año de nuestro estudio, se descartaba puesto que no se podía analizar su evolución. Para el resto de las empresas se consideraron tres posibles comportamientos: (a) estable (si una vez conseguido el certificado se mantenía en el resto de los años del estudio -fidelidad completa-); (b) intermitente (si se abandonaba en algún periodo, pero, posteriormente, se volvía a obtener -fidelidad parcial-); y (c) abandono (una vez conseguido el certificado se mantenía hasta abandonarlo definitivamente -sin fidelidad-). 
Finalmente, se llevó a cabo un estudio de posibles relaciones de dependencia entre variables. Concretamente, se contrastaron las hipótesis planteadas utilizado el estadístico de la Chi-Cuadrado y el análisis discriminante mediante el programa estadístico SPSS.

\section{RESULTADOS}

\subsection{Certificaciones ISO 9001 y "Q de calidad" por subsectores}

Los resultaron obtenidos evidenciaron, en primer lugar, que, en el periodo comprendido entre 2006 y 2018, de los 603 establecimientos analizados, 300 dispusieron en algún momento de la "Q de calidad" (49,8\%), 226 de la ISO 9001 (37,5\%) y 77 habían optado por tener ambos certificados, bien en el mismo año o en diferentes años (12,7\%) (Tabla 3$)$.

Es reseñable que los establecimientos de catering no utilizaron la " $Q$ de calidad", mientras que de los 304 restaurantes, bares y cafeterías que dispusieron de uno de estos sellos en el periodo de estudio, 223 sí la eligieron, frente a los 102 que optaron por la ISO 9001, sola o conjuntamente con la " $Q$ de calidad".

Tabla 3. Disponibilidad de certificaciones ISO 9001 y “Q de calidad" en el periodo 2006 a 2018, según tipo de establecimiento

\begin{tabular}{|c|c|c|c|c|c|}
\hline \multirow{2}{*}{$\begin{array}{l}\text { Tipo de estableci- } \\
\text { miento }\end{array}$} & \multirow[b]{2}{*}{$\begin{array}{c}\text { Solo ISO } \\
9001\end{array}$} & \multirow{2}{*}{$\begin{array}{l}\text { Solo "Q de } \\
\text { calidad" }\end{array}$} & \multicolumn{2}{|c|}{ ISO 9001 y “Q de calidad" } & \multirow[b]{2}{*}{ TOTAL } \\
\hline & & & Simultáneamente & $\begin{array}{l}\text { Diferentes } \\
\text { años }\end{array}$ & \\
\hline Apartamentos & $6(28,6 \%)$ & $11(52,4 \%)$ & $3(14,3 \%)$ & $1(4,8 \%)$ & 21 \\
\hline Hoteles & $108(43,7 \%)$ & $87(35,2 \%)$ & $44(17,8 \%)$ & $8(3,2 \%)$ & 247 \\
\hline $\begin{array}{l}\text { Restaurantes, bares y } \\
\text { cafeterías }\end{array}$ & $81(26,6 \%)$ & $202(66,4 \%)$ & $19(6,3 \%)$ & $2(0,7 \%)$ & 304 \\
\hline Catering & $31(100 \%)$ & $0(0 \%)$ & $0(0 \%)$ & $0(0 \%)$ & 31 \\
\hline TOTAL & $226(37,5 \%)$ & $300(49,8 \%)$ & $66(10,9 \%)$ & $11(1,8 \%)$ & 603 \\
\hline
\end{tabular}

Fuente: elaborado a partir de Junta de Andalucía (2007 a 2019).

Por tipo de establecimiento fueron los hoteles los más propensos a utilizar las dos certificaciones, con más del $21 \%$. En concreto, el $17,8 \%$ de los hoteles dispusieron de los sellos simultáneamente, mientras que el 3,2\% restante en diferentes años.

El contraste de hipótesis con el estadístico de la Chi-Cuadrado confirmó las diferencias entre los dos grandes subsectores de alojamiento (apartamentos y hoteles) y restauración (restaurantes, bares, cafeterías y catering), tanto en los establecimientos que se certificaron solo de ISO $9001\left(\chi^{2}=5,267, p=0,022\right)$ como en los que optaron únicamente por la " $Q$ de calidad" $\left(\chi^{2}=33,541, p=0,000\right)$. Además, la utilización de ISO 9001 y " $Q$ de calidad" por un mismo establecimiento sí dependía del subsector $\left(\chi^{2}=28,597, p=0,000\right)$, siendo más habitual esta situación en los alojamientos. 
Por otro lado, el estudio de la proporción, mediante la técnica de análisis discriminante, nos indicó, con un nivel de confianza del $90 \%(\alpha=0,1)$, que existía una tendencia a obtener la certificación ISO 9001 en el sector de los alojamientos, por encima de la certificación " $Q$ de calidad", mientras que los establecimientos de restauración tenían la predisposición contraria (Tabla 4).

Tabla 4. Análisis discriminante de las certificaciones ISO 9001 y "Q de calidad” (alojamiento o restauración)

\begin{tabular}{|c|c|c|c|c|c|}
\hline & \multirow{2}{*}{\multicolumn{2}{|c|}{ Alojamiento }} & \multirow{2}{*}{\multicolumn{2}{|c|}{ Restauración }} \\
\hline & & & & & \\
\hline & & ISO 9001 & "Q de calidad" & ISO 9001 & "Q de calidad" \\
\hline \multicolumn{2}{|r|}{ Proporción con certificado (p) } & $63,4328 \%$ & $57,4627 \%$ & $39,7015 \%$ & $66,5672 \%$ \\
\hline \multicolumn{2}{|r|}{ Proporción sin certificado (q) } & $36,5672 \%$ & $42,5373 \%$ & $60,2985 \%$ & $33,4328 \%$ \\
\hline \multicolumn{2}{|r|}{ Tamaño muestral (n) } & \multicolumn{2}{|r|}{268} & \multicolumn{2}{|r|}{335} \\
\hline \multirow{3}{*}{ 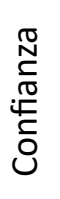 } & Nivel & \multicolumn{2}{|r|}{$90 \%$} & \multicolumn{2}{|c|}{$90 \%$} \\
\hline & Límite inferior del intervalo & $59,66 \%$ & $53,59 \%$ & $36,28 \%$ & $63,26 \%$ \\
\hline & Límite superior del intervalo & $67,20 \%$ & $61,33 \%$ & $43,13 \%$ & $69,87 \%$ \\
\hline \multirow{2}{*}{ 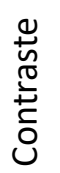 } & Estadístico T & \multicolumn{2}{|c|}{1,4160} & \multicolumn{2}{|c|}{7,2347} \\
\hline & $\mathrm{p}$ valor & \multicolumn{2}{|c|}{0,0784} & \multicolumn{2}{|c|}{0,0000} \\
\hline
\end{tabular}

Centrándonos en la evolución de estas certificaciones en el período analizado (Gráfico 5), podemos observar cómo en el año 2012 se produjo un gran pico de empresas que abandonaron la ISO 9001, lo cual fue compensado, en parte, por la entrada de nuevos establecimientos. Esta situación se debió la salida de un gran número de hoteles, entre los cuales se encontraban la mayoría de los pertenecientes a la cadena Paradores. Esta reducción estaba precedida de un aumento en el número de nuevas entradas en la " $Q$ de calidad" el año anterior. Sin embargo, el análisis individualizado de las empresas que abandonaron la ISO en 2012 indicó que no cambiaron de certificado. Posteriormente, en 2016, el elevado número de empresas salientes de ISO 9001 no fue compensado por las entrantes, originando un desplome del total de certificados hasta 2018.

Por lo que respecta a la " $Q$ de calidad" (Gráfico 5), el mayor número de abandonos se produjo en 2017 y fue originado por la decisión de la cadena de restaurantes McDonald's de no mantener dicho sello, lo cual supuso una reducción de 92 establecimientos. 
Gráfico 5. Evolución de las certificaciones ISO 9001 y "Q de calidad" de los establecimientos hoteleros y de restauración de Andalucía

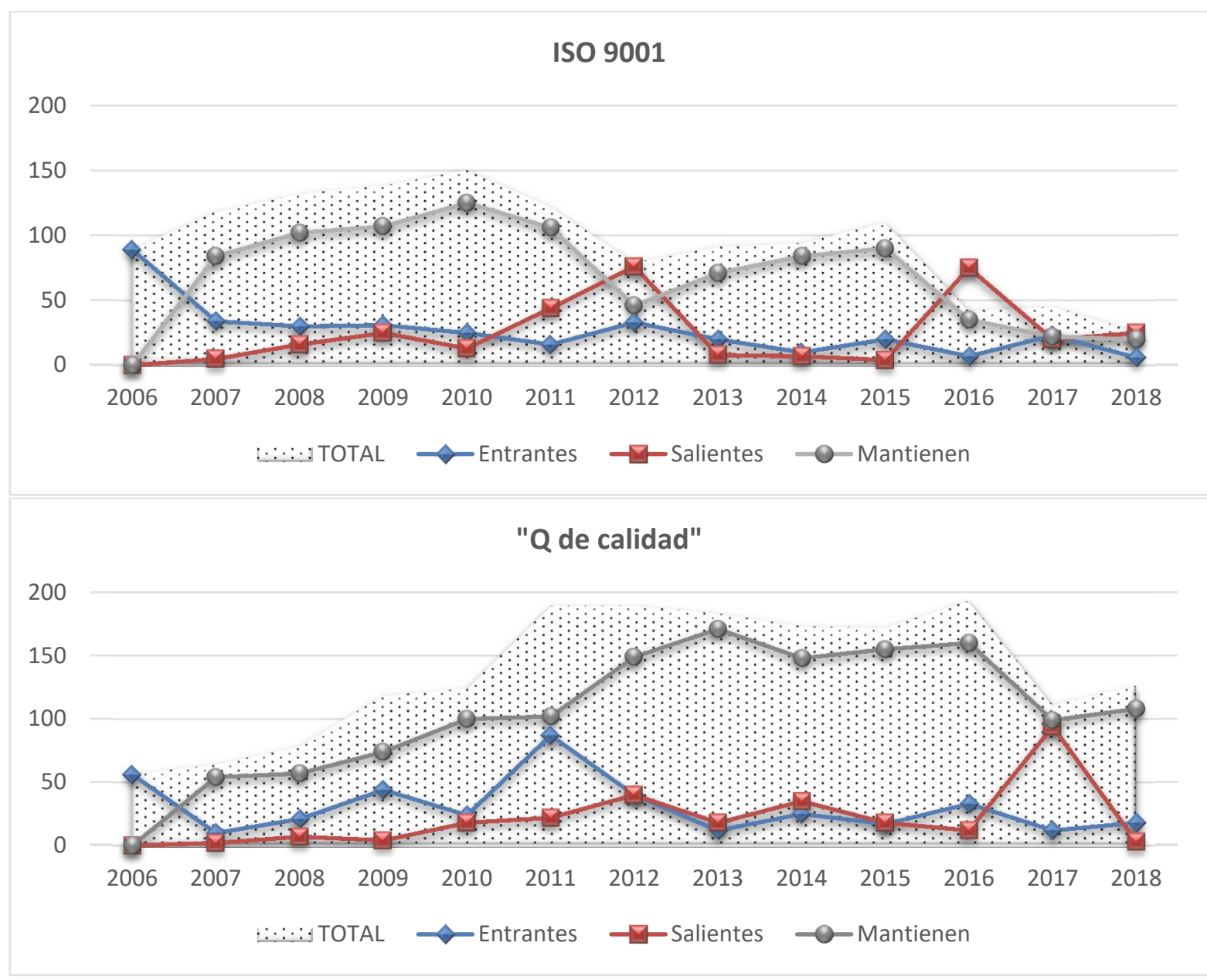

Fuente: elaborado a partir de Junta de Andalucía (2007 a 2019).

\subsection{Fidelidad a los certificados}

Para el análisis de la fidelidad se eliminaron del total de establecimientos analizados aquellos que obtuvieron la certificación por primera vez en 2018 y que, por tanto, no podían ser estudiados con posterioridad ( 3 empresas que obtuvieron la ISO 9001 y 15 que consiguieron la "Q de calidad" en 2018). Siguiendo el criterio de empresa "estable o con fidelidad completa", "intermitente o fidelidad parcial", y que "abandonaba" el certificado para no volverlo a obtener en el período de estudio (sin fidelidad), se construyó la Tabla 5.

Los resultados evidenciaron un comportamiento más estable en las empresas que disponían de la " $Q$ de calidad" (Tabla 5) existiendo una clara diferencia entre el número de establecimientos que mantuvieron dicho certificado durante el periodo de estudio $(28,18 \%)$ frente a los que lo hicieron con la ISO 9001 (4,33\%). Con respecto al abandono de los certificados, el $82,67 \%$ de las empresas que dispusieron del certificado ISO 9001 decidieron no renovarlo pasado un periodo de tiempo, siendo especialmente significativo el caso de los apartamentos y empresas de catering $(90,32 \%$ y $90 \%$ respectivamente). Sin embargo, el porcentaje 
de abandono de la " $Q$ de calidad" fue sensiblemente menor $(65,75 \%)$, lo que refleja una mayor fidelidad a este certificado. En conjunto, de los 486 abandonos de los certificados, solamente 22 casos de ISO 9001 y 4 casos de "Q de calidad" estuvieron totalmente justificados por el cierre de la actividad.

Tabla 5. Número de establecimientos hoteleros y de restauración de Andalucía por tipo y comportamiento ante las certificaciones ISO 9001 y " $Q$ de calidad"

\begin{tabular}{|c|c|c|c|c|c|}
\hline & & \multicolumn{3}{|c|}{ Tipo de comportamiento } & \multirow{2}{*}{ Total } \\
\hline \multicolumn{2}{|c|}{ Tipo de establecimiento } & Estable & Intermitente & Abandono & \\
\hline \multirow{5}{*}{ ISO 9001} & Apartamentos & $0(0,00 \%)$ & $1(10,00 \%)$ & $9(90,00 \%)$ & 10 \\
\hline & Hoteles & $3(1,89 \%)$ & $21(13,21 \%)$ & $135(84,91 \%)$ & 159 \\
\hline & Rest. bares y cafeterías & $8(8,00 \%)$ & $16(16,00 \%)$ & $76(76,00 \%)$ & 100 \\
\hline & Catering & $2(6,45 \%)$ & $1(3,23 \%)$ & $28(90,32 \%)$ & 31 \\
\hline & Total ISO 9001 & $13(4,33 \%)$ & $39(13,00 \%)$ & $248(82,67 \%)$ & 300 \\
\hline \multirow{5}{*}{ "Q de calidad" } & Apartamentos & $4(26,67 \%)$ & $3(20,00 \%)$ & $8(53,33 \%)$ & 15 \\
\hline & Hoteles & $40(28,99 \%)$ & $8(5,80 \%)$ & $90(65,22 \%)$ & 138 \\
\hline & Rest., bares y cafeterías & $58(27,75 \%)$ & $11(5,26 \%)$ & $140(66,99 \%)$ & 209 \\
\hline & Catering & $0(0,00 \%)$ & $0(0,00 \%)$ & $0(0,00 \%)$ & 0 \\
\hline & Total “Q de calidad” & $102(28,18 \%)$ & $22(6,08 \%)$ & $238(65,75 \%)$ & 362 \\
\hline
\end{tabular}

Fuente: elaborado a partir de Junta de Andalucía (2007 a 2019).

De este modo, la fidelidad completa se dio en un mayor grado en los establecimientos de restauración, para la ISO 9001, y en los restaurantes, bares y cafeterías para la " $Q$ de calidad". La fidelidad parcial de los establecimientos fue bastante baja en el caso de la " $Q$ de calidad" (6,08\%, en términos globales), lo que parece reflejar que cuando se abandona este sello es menos habitual darle una segunda oportunidad, a diferencia de lo que se muestra en la ISO 9001 (13\% de media). Además, un análisis más individualizado de los datos nos mostró que, entre los casos que simultanean las dos certificaciones, es más habitual que abandonen la ISO 9001 que la "Q de calidad".

Completando este análisis con el número de años que mantenían los certificados las empresas (Gráfico 6), podemos resaltar que los hoteles y apartamentos fieles a las certificaciones " $Q$ de calidad" (fidelidad completa o parcial) las mantuvieron, de media, un mayor número de años que los que fueron fieles a la ISO 9001. En concreto, en el caso de los hoteles con comportamiento estable, la " $Q$ de calidad" se mantuvo durante 10,2 años (con un rango que iba desde un mínimo de 2 años y un máximo de 13, es decir todo el periodo de estudio) y 7,5 años cuando el comportamiento fue intermitente; mientras que la ISO 9001, en esta tipología de establecimiento, se mantuvo, de media durante 3,67 años, cuando se consideró fidelidad completa o comportamiento estable, y un total de 6 años cuando fue intermitente. El caso de los apartamentos es aún más llamativo, ya que no se halló ningún caso de comportamiento 
estable en la ISO 9001, mientras que, de media, los que fueron fieles a la "Q de calidad", la mantuvieron durante 9,5 años.

Gráfico 6. Valores medios, máximos y mínimos del número de años certificados en ISO 9001 y "Q de calidad", según tipo de establecimiento y tipo de comportamiento

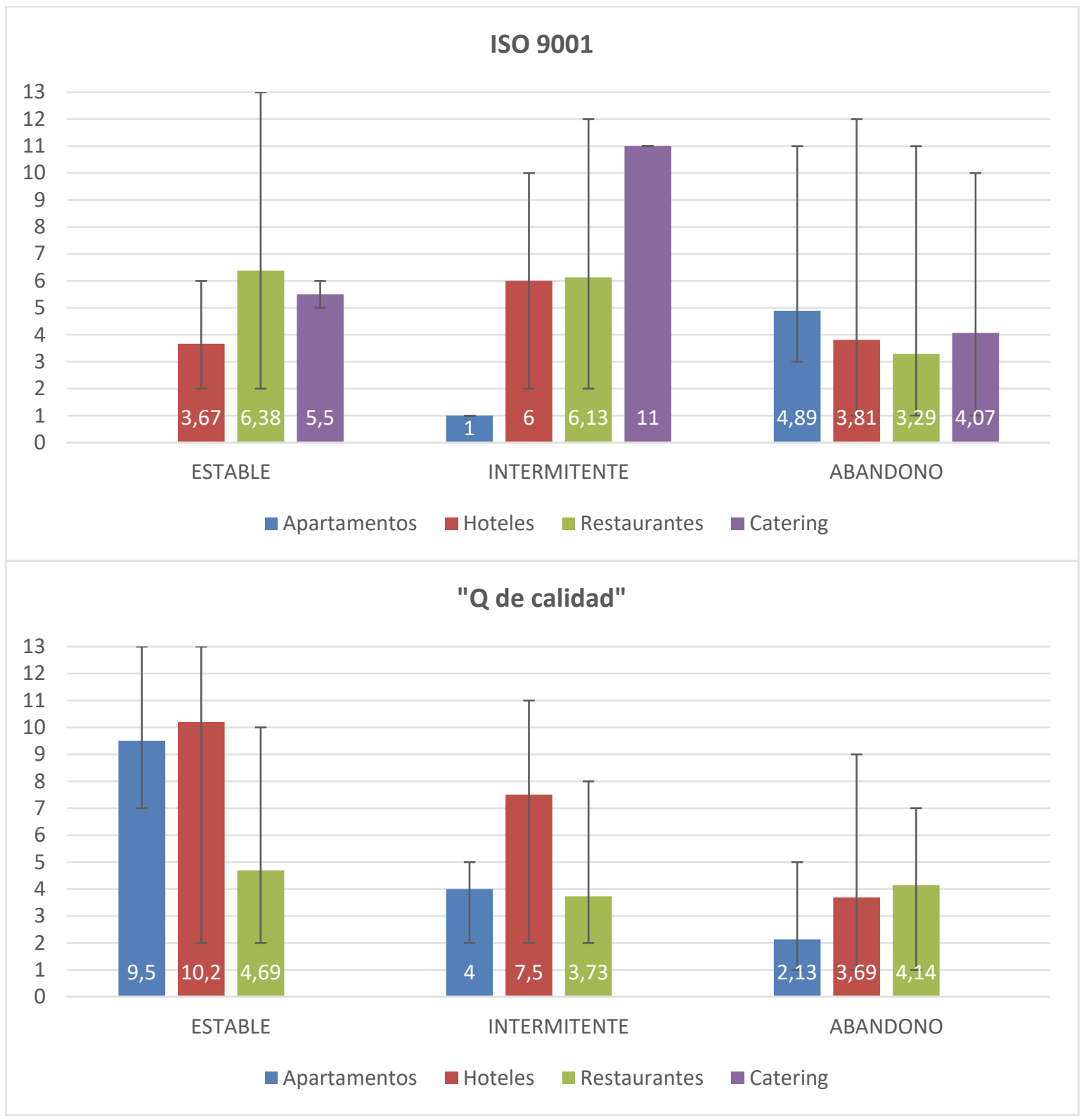

Fuente: elaborado a partir de Junta de Andalucía (2007 a 2019).

Finalmente, se demostró que la fidelidad dependía del tipo de establecimiento. El estadístico de la Chi-Cuadrado reflejó la existencia de una relación estadísticamente significativa en el caso de la ISO $9001\left(\chi^{2}=6,147, p=0,046\right)$, si bien no se encontraron diferencias en el comportamiento de los establecimientos que obtuvieron el sello " $Q$ de calidad" $\left(\chi^{2}=0,687, p\right.$ $=0,709)$. 
En este sentido, el estudio de la proporción, mediante la técnica de análisis discriminante (Tabla 6), nos mostró que la fidelidad a la " $Q$ de calidad" era mucho mayor que a la ISO 9001 , tanto en los alojamientos como en restauración $(\alpha=0,1)$.

Tabla 6. Análisis discriminante de la fidelidad a certificaciones ISO 9001 y “Q de calidad” (alojamiento o restauración)

\begin{tabular}{|c|c|c|c|c|c|}
\hline & & \multicolumn{2}{|c|}{ Alojamiento } & \multicolumn{2}{|c|}{ Restauración } \\
\hline & & ISO 9001 & "Q de calidad" & ISO 9001 & "Q de calidad" \\
\hline \multicolumn{2}{|r|}{ Proporción con certificado ( $p$ ) } & $1,7751 \%$ & $28,7581 \%$ & $7,6336 \%$ & $27,7752 \%$ \\
\hline \multicolumn{2}{|r|}{ Proporción sin certificado (q) } & $98,2248 \%$ & $71,2418 \%$ & $92,3664 \%$ & $72,2488 \%$ \\
\hline \multicolumn{2}{|r|}{ Tamaño muestral (n) } & 169 & 153 & 131 & 209 \\
\hline \multirow{3}{*}{ 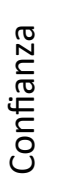 } & Nivel & \multicolumn{2}{|r|}{$90 \%$} & \multicolumn{2}{|c|}{$90 \%$} \\
\hline & Límite inferior del intervalo & $0,47 \%$ & $24,07 \%$ & $4,66 \%$ & $23,78 \%$ \\
\hline & Límite superior del intervalo & $3,08 \%$ & $33,45 \%$ & $10,61 \%$ & $31,72 \%$ \\
\hline \multirow{2}{*}{ 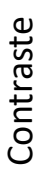 } & Estadístico T & \multicolumn{2}{|c|}{7,1051} & \multicolumn{2}{|c|}{5,1985} \\
\hline & $p$ valor & \multicolumn{2}{|c|}{0,0000} & \multicolumn{2}{|c|}{0,0000} \\
\hline
\end{tabular}

La Tabla 7 muestra, a modo de resumen, los resultados del contraste de hipótesis, relacionándolas con las preguntas de investigación, donde se puede apreciar que, excepto para la fidelidad de la " $Q$ de calidad", el tipo de establecimiento afecta tanto a la elección de las certificaciones analizadas como en la fidelidad a la ISO 9001.

Tabla 7. Preguntas de investigación, hipótesis y resultados del contraste

\begin{tabular}{|c|c|c|}
\hline Pregunta de investigación & Hipótesis & Resultado \\
\hline \multirow{2}{*}{$\begin{array}{l}\text { ¿Hay diferencias significativas } \\
\text { en la elección de las certifi- } \\
\text { caciones ISO } 9001 \text { y “Q de } \\
\text { calidad" según el subsector, } \\
\text { dentro del alojamiento y res- } \\
\text { tauración? }\end{array}$} & $\begin{array}{l}\text { H1a: En los alojamientos hay diferencias } \\
\text { significativas entre la proporción de certifi- } \\
\text { cación de ISO } 9001 \text { y "Q de calidad" }\end{array}$ & $\begin{array}{l}\text { Confirmada (mayor } \\
\text { proporción de ISO } \\
\text { 9001) } \\
\end{array}$ \\
\hline & $\begin{array}{l}\text { H1b: En la restauración hay diferencias } \\
\text { significativas entre la proporción de certifi- } \\
\text { cación de ISO } 9001 \text { y "Q de calidad" }\end{array}$ & $\begin{array}{l}\text { Confirmada (mayor } \\
\text { proporción de "Q } \\
\text { de calidad") }\end{array}$ \\
\hline $\begin{array}{l}\text { ¿Hay tipos de establecimientos } \\
\text { más propensos a utilizar las } \\
\text { dos certificaciones }\end{array}$ & $\begin{array}{l}\text { H1c: La elección conjunta de ISO } 9001 \text { y "Q } \\
\text { de calidad" está relacionada con el tipo de } \\
\text { establecimiento }\end{array}$ & $\begin{array}{l}\text { Confirmada (mayor } \\
\text { uso conjunto en } \\
\text { alojamiento) }\end{array}$ \\
\hline \multirow{2}{*}{$\begin{array}{l}\text { ¿Existen diferencias de fideli- } \\
\text { dad a certificaciones ISO } 9001 \\
\text { y "Q de calidad" entre los } \\
\text { diferentes tipos de estableci- } \\
\text { mientos dentro del sector de } \\
\text { hoteles y restauración? }\end{array}$} & $\begin{array}{l}\text { H2a: El nivel de fidelidad de los alojamien- } \\
\text { tos a la ISO } 9001 \text { es diferente al alcanzado } \\
\text { con la "Q de calidad" }\end{array}$ & $\begin{array}{l}\text { Confirmada (mayor } \\
\text { fidelidad a "Q de } \\
\text { calidad") }\end{array}$ \\
\hline & $\begin{array}{l}\text { H2b: El nivel de fidelidad de la restauración } \\
\text { a la ISO } 9001 \text { es diferente al alcanzado con } \\
\text { la "Q de calidad" }\end{array}$ & $\begin{array}{l}\text { Confirmada (mayor } \\
\text { fidelidad a "Q de } \\
\text { calidad") }\end{array}$ \\
\hline
\end{tabular}




\section{CONCLUSIONES Y DISCUSIÓN DE RESULTADOS}

En el periodo de estudio los establecimientos hoteleros y de restauración andaluces apostaron mayoritariamente por la " $Q$ de calidad", manteniéndola activa una media de 4,7 años. Si bien tan solo el 34\% de los que dispusieron de este sello en el periodo 2006-2018 renovaron la certificación, compensándose los nuevos establecimientos certificados con los que abandonaban. En este sentido, desde 2009 a 2018, la "Q de calidad" en este sector parece haber evolucionado de una etapa de madurez a una de declive, registrando, en 2017, una caída del $42 \%$ de las certificaciones respecto a 2016, tanto en España como en Andalucía. Esta caída, contrasta con el pronóstico realizado por Alonso-Almeida et al. (2013), en el que consideraron que la " $Q$ de calidad" crecería en España hasta 2019, momento en que alcanzaría el punto de saturación y dejaría de crecer.

En cuanto a la ISO 9001, la fidelidad baja al $17 \%$ y el declive es más que evidente, coincidiendo con las conclusiones de otros estudios (Alonso-Almeida et al., 2013; Djofack y Robledo, 2017). Además, se comprobó que, si un establecimiento se decantaba solo por una de las certificaciones, los alojamientos elegían la ISO 9001, mientras que la restauración optaba por la "Q de calidad".

La mayor inclinación hacia la " $Q$ de calidad" del sector turístico se asimila a la obtenida en otras regiones españolas (Ballina y Valdés, 2016; Djofack y Robledo, 2017) y puede ser debida a que "la interiorización de los requisitos de la marca " $Q$ de calidad" tiene beneficios para los clientes, empleados y sociedad, además de para la organización" (Pereira-Moliner et al., 2016) o a la mejora percibida por los clientes, a través de la mejor puntuación que éstos otorgan una vez disfrutada la experiencia del servicio en esta región (Fuentes, et al., 2016). Esto está en consonancia con la mayor fidelidad a la "Q de calidad" que a la ISO 9001, tanto en alojamiento como en restauración. Incluso, se ha observado que un $10,9 \%$ de los establecimientos, mayoritariamente hoteleros, optó por simultanear este certificado con el de la ISO 9001, quizá motivados por las expectativas de mejora en los resultados financieros y comerciales, tal y como se recoge en el estudio de Ballina y Valdés (2016).

Por otro lado, en relación con la fidelidad, a la ISO 9001, esta ha quedado reducida a mínimos, habiendo demostrado, además, que es muy inferior a la que obtiene la " $Q$ de calidad" tanto en los alojamientos como en la restauración.

El abandono de estos certificados puede deberse a que los establecimientos consideren que disponer y publicitar las certificaciones de calidad no actúe ya como una señal que atraiga a los consumidores y, por tanto, que su utilización no sea rentable. En esta línea, HerasSaizarbitoria (2011), indica que esta cuestión puede estar relacionada con la posible erosión del prestigio y la credibilidad de los metaestándares. También queda patente que la causa de la descertificación no ha sido un trasvase de la ISO 9001 hacia la "Q de calidad", o viceversa, por lo que los establecimientos hoteleros y de restauración pueden estar manteniendo los procedimientos de los sistemas de gestión sin volverse a certificar, lo que se incluye entre los motivos planteados por Zimon y Dellana (2019) para abandonar un certificado.

En este sentido, los establecimientos pueden verse más atraídos por publicitar las opiniones online o electronic Word of Mouth (eWOM), que actúan como potentes señales y 
suponen una influencia, especialmente importante, en el sector del turismo y alojamiento. Si bien la reputación online puede ser el reflejo del nivel de satisfacción de los clientes y resultado del sistema de gestión de calidad del establecimiento, una vez publicada puede actuar como señal de calidad y repercutir en los resultados económicos de los establecimientos (Zhao et al., 2015; Diana-Jens y Rodríguez, 2015; Ogut y Tas, 2012; Tsao et al., 2015), suponiendo su gestión unos costes mucho menores que los que implica la certificación. No obstante, certificados y reputación online se plantean como complementarios ya que, si bien el proceso de certificación puede proveer a los establecimientos de un sistema de calidad que mejore sus procesos, la utilidad del mismo como señal de calidad para los clientes puede estar siendo sustituida por la reputación online. A este respecto, según el último barómetro de TripAdvisor (2018), el 86\% de los encuestados no reservaría un alojamiento sin haber leído previamente opiniones relevantes sobre el mismo. En esta línea, el estudio de Autor et al. (2019), realizado sobre las señales de calidad emitidas por los alojamientos rurales andaluces en sus páginas web oficiales, el $23,68 \%$ de los establecimientos mostró información relacionada con su reputación online, frente al 7,5\% que mostraban sus certificaciones de calidad.

Si a la baja fidelidad observada en nuestro estudio se une el impacto de la COVID-19, es probable que la cifra de abandonos se acreciente por la imposibilidad de afrontar, entre otros, los costes asociados a la renovación de la certificación, así como por el cierre definitivo de establecimientos.

Desde el punto de vista teórico, este trabajo profundiza en los resultados de Casadesús (2010) y Alonso-Almeida et al. (2013), poniendo el foco en las variables componentes del volumen de certificaciones (nuevas certificaciones, renovación y abandono). De esta forma, presentamos un primer estudio que arroja luz sobre la fidelidad a la ISO 9001 y " $Q$ de calidad", mostrando diferencias de comportamiento en los establecimientos de alojamiento y los de restauración, lo cual puede ayudar a orientar más adecuadamente las estrategias de difusión de estas certificaciones.

Consideramos, al igual que en el trabajo de Heras-Saizarbitoria (2011), que los resultados expuestos pueden ser interesantes para los organismos nacionales e internacionales promotores y prescriptores de estos estándares e incluso para las propias administraciones públicas, que deberían hacer una reflexión serena y rigurosa sobre el fenómeno de las certificaciones y evitar que se produzca una excesiva confusión y saturación. En este sentido, en términos generales, los altos porcentajes de abandono nos llevan a aconsejar mejorar la fidelidad mediante reducciones de costes de mantenimiento, ya que si consideramos que en la decisión de certificarse priman los motivos internos sobre los externos (Djofack y Robledo, 2017; Álvarez et al., 2012; Claver et al., 2006), una vez que han hecho los cambios organizativos necesarios para obtener certificado, los establecimientos pueden mantener el sistema de calidad sin el sello.

Por otro lado, nos planteamos una serie de consideraciones a tener en cuenta por los establecimientos hoteleros y de restauración que se planteen obtener una de estas certificaciones: 
- Para los hoteles, es importante resaltar la importancia que puede tener la aprobación de la norma "UNE-ISO 22483:2020", que anula la que regulaba la correspondiente a los requisitos para obtener la certificación " $Q$ de calidad", ya que se presenta como una mejora en relación a la internacionalización, pudiendo aumentar su atractivo de cara a una posible implementación, convirtiéndose en un sustituto de la ISO 9001.

- Para el caso concreto de la restauración, si seguimos la tendencia del sector, sería más aconsejable la elección de la " $Q$ de calidad" que la ISO 9001, ya que existe una mayor propensión a la elección de la primera, lo que puede deberse a la adecuación de este tipo de certificación a las características propias de este subsector.

- Además, en todos los casos es necesario considerar que, aunque es imprescindible estandarizar los procesos para reducir errores y mejorar la calidad del servicio y la toma de decisiones, las motivaciones externas relativas a la mejora de la imagen por parte de los clientes se está viendo sustituida por la reputación online, que está desplazando a las certificaciones de calidad como señales de prestigio.

En relación con las limitaciones del estudio, es necesario aclarar que, para incluir todos los establecimientos de alojamiento, sería preciso incorporar los datos de albergues, alojamientos rurales y campings. No obstante, la revisión de los "Directorios de Establecimientos y Espacios Certificados" (Junta de Andalucía, 2007-2019) nos permite intuir que son muy pocas las empresas de ese tipo que dispusieron de ISO 9001 y “Q de calidad" en el periodo de estudio.

Por último, somos conscientes que los resultados de este estudio se centran en Andalucía y a un periodo de tiempo limitado, por lo que no se pueden extrapolar al resto de España. Sin embargo, el análisis de la fidelidad a las certificaciones podría aplicarse a cualquier otra región y sector de actividad, pudiendo proporcionar información muy útil sobre la compensación del abandono de certificados para poder tomar medidas para fidelizar a las empresas. Por ello, para complementar este trabajo se pretende realizar un proyecto de investigación que incluya los motivos de pérdida de certificaciones, encuestando a los establecimientos que no renovaron su certificación.

En este sentido, entre las futuras líneas de investigación se encuentra replicar este estudio en otras comunidades autónomas, para comparar sus resultados con Andalucía. Además, también se encuentra entre nuestros proyectos realizar un análisis similar con otras certificaciones o distinciones dentro de la misma comunidad autónoma. 


\section{REFERENCIAS BIBLIOGRÁFICAS}

Akerlof, G. A. (1970). The Market for "Lemons": Quality Uncertainty and the Market Mechanism. The Quarterly Journal of Economics, 84(3), 488-500. https://doi.org/10.2307/1879431

Alič, M. (2014). Impact of ISO 9001 certification cancellation on business performance: a case study in Slovenian organisations. Total Quality Management and Business Excellence, 25(7-8), 790-811. https://doi.org/10.1080/14783363.2014.906107

Alonso-Almeida, M. del M. y Rodríguez-Antón, J. M. (2011). Organisational behaviour and strategies in the adoption of certified management systems: An analysis of the Spanish hotel industry. Journal of Cleaner Production, 19(13), 1455-1463. https://doi.org/10.1016/i. jclepro.2011.04.015

Alonso-Almeida, M. del M., Marimon, F. y Bernardo, M. (2013). Diffusion of quality standards in the hospitality sector. International Journal of Operations \& Production Management, 33(5), 504-527. https://doi.org/10.1108/01443571311322706

Alonso-Almeida, M. del M., Rodríguez-Antón, J. M. y Rubio-Andrada, L. (2012). Reasons for implementing certified quality systems and impact on performance: An analysis of the hotel industry. Service Industries Journal, 32(6), 919-936. https://doi.org/10.1080/026 $\underline{42069.2010 .545886}$

Álvarez García, J., Fraiz Brea, J. A. y del Rio Rama, M. de la C. (2012). Análisis de las motivaciones para certificar la marca " $Q$ de calidad turística". Sector de alojamiento. Investigaciones Europeas de Direccion y Economia de La Empresa, 18(1), 101-121. https://doi.org/10.1016/S1135-2523(12)60063-X

Álvarez García, J., Fraiz Brea, J. A. y del Río Rama, M. de la C. (2013). Alojamiento Rural. Barreras a la implantación y certificación de la " $Q$ de Calidad Turística." Revista de Análisis Turistico, 15, 13-24. https://doi.org/http://www.aecit.org/jornal/index.php/ AECIT/article/view/140

Álvarez-García, J., del Río-Rama, M. de la C. y Biagio, S. (2017). Quality management and customer results: the tourist accommodation sector in Spain. International Journal of Quality \& Reliability Management, 34(5), 701-719. https://doi.org/http://dx.doi.org/10.1108/ MRR-09-2015-0216

Ballina Ballina, F. J. y Valdés Peláez, L. (2016). Los efectos de la combinación de estándares de calidad sobre los resultados empresariales en el sector hotelero. Universia Business Review, 2016(52), 198-221. https://doi.org/10.3232/UBR.2016.V13.N4.05

Ballina, F. J., Valdés, L. y Del Valle, E. (2019). The Signalling Theory: The Key Role of Quality Standards in the Hotels Performance. Journal of Quality Assurance in Hospitality and Tourism, 21(15), 1-19. https://doi.org/10.1080/1528008X.2019.1633722

Boulding, W. y Kirmani, A. (1993). A Consumer-Side Experimental Examination of Signaling Theory: Do Consumers Perceive Warranties as Signals of Quality? Journal of Consumer Research, 20(1), 111-123. https://doi.org/10.1086/209337

Cabecinhas, M., Domingues, P., Sampaio, P., Bernardo, M., Franceschini, F., Galetto, M. y Hernandez-Vivanco, A. (2018). Integrated management systems diffusion models in South European countries. International Journal of Quality and Reliability Management, 35(10), 2289-2303. https://doi.org/10.1108/IJQRM-03-2017-0044 
Calderwood, L. U., Soshkin, M. \& Fisher, M. (2019). The Travel \& Tourism Competitiveness Report 2019: Travel and Tourism at a Tipping Point. World Economic Forum. http:// www3.weforum.org/docs/WEF_TTCR_2019.pdf

Cândido, C. J. F., Coelho, L. M. S. y Peixinho, R. M. T. (2016). The Financial Impact of a Withdrawn ISO 9001Certificate. International Journal of Operations \& Production Management, 36(1), 23-41.

Cândido, C. J. F., Coelho, L. M. S. y Peixinho, R. M. T. (2019). Why firms lose their ISO 9001 certification: Evidence from Portugal. Total Quality Management and Business Excellence, 32:5-6, 632-651. https://doi.org/10.1080/14783363.2019.1625266

Casadesús, M. y Karapetrovic, S. (2005). The erosion of ISO 9000 benefits: A temporal study. International Journal of Quality and Reliability Management, 22(2), 120-136. https:// doi.org/10.1108/02656710510577198

Casadesús, M., Marimon, F. y Alonso, M. (2010). The future of standardised quality management in tourism: Evidence from the spanish tourist sector. Service Industries Journal, 30(14), 2457-2474. https://doi.org/10.1080/02642060802712822

Claver, E., Tarí, J. J. y Pereira, J. (2006). Does quality impact on hotel performance? International Journal of Contemporary Hospitality Management, 18(4), 350-358. https://doi. org/10.1108/09596110610665357

Connelly, B. L., Certo, S. T., Ireland, R. D. y Reutzel, C. R. (2011). Signaling theory: A review and assessment. Journal of Management, 37(1), 39-67. https://doi. org/10.1177/0149206310388419

Diana-Jens, P. y Rodríguez Ruibal, A. (2015). La reputación online y su impacto en la política de precios de los hoteles. Cuadernos de Turismo, (36), 129-155. https://doi.org/10.6018/ turismo.36.230911

Djofack, S. y Robledo Camacho, M. A. (2017). Implementation of ISO 9001 in the Spanish tourism industry. International Journal of Quality \& Reliability Management, 34(1), 18-37. https://doi.org/10.1108/09574090910954864

Franceschini, F., Galetto, M., Maisano, D. y Mastrogiacomo, L. (2010). Clustering of European countries based on ISO 9000 certification diffusion. International Journal of Quality and Reliability Management, 27(5), 558-575. https://doi.org/10.1108/02656711011043535

Fuentes Medina, M. L., Hernández Estárico, E. y Morini Marrero, S. (2016). Q de calidad y satisfacción del turista en el sector hotelero español. Cuadernos de Turismo, (37), 203-226. https://doi.org/10.6018/turismo.37.256211

Gianni, M. y Gotzamani, K. (2015). Management systems integration: Lessons from an abandonment case. Journal of Cleaner Production, 86, 265-276. https://doi.org/10.1016/i. jclepro.2014.08.023

Heras-Saizarbitoria, I. (2011). ¿Qué fue de la isomanía? ISO 9000, ISO 14000 y otros metaestándares en perspectiva. Universia Business Review, 1(29), 66-79.

Heras-Saizarbitoria, I., Arana, G. y Boiral, O. (2015). Do ISO 9001-certified hotels get a higher customer rating than non-certified ones? International Journal of Hospitality Management, 51, 138-146. https://doi.org/10.1016/i.ijhm.2015.08.006

ICTE Instituto para la Calidad Turística Española. (2019). Q de calidad Comparativa Evolución Sectores 2018. 
IECA Instituto de Estadística y Cartografía de Andalucía (2019) Directorio de Establecimientos y Empresas con actividad económica en Andalucía. https://www.juntadeandalucia.es/institutodeestadisticaycartografia/badea/operaciones/consulta/ anual $/ 210$ ?CodOper $=$ b3 $131 \&$ codConsulta $=210$

IECA Instituto de Estadística y Cartografía de Andalucía (2020) Encuesta de Coyuntura Turística de Andalucía (ECTA).https://www.juntadeandalucia.es/institutodeestadisticaycartografia/turismo/index.htm

Iglesias, V., De la Ballina, F. J. y Caso, L. (2018). Investment decisions on quality certifications by hotel chains: Differences between ex-ante and ex-post decisions. Spanish Journal of Marketing - ESIC, 22(1), 83-102. https://doi.org/10.1108/SJME-03-2018-001

Ikram, M., Zhang, Q. y Sroufe, R. (2020). Future of quality management system (ISO 9001) certification: novel grey forecasting approach. Total Quality Management and Business Excellence. https://doi.org/10.1080/14783363.2020.1768062

INE Instituto Nacional de Estadística (2020). Movimientos Turísticos en Fronteras 2020. Madrid: Instituto Nacional de Estadística. https://www.ine.es/jaxiT3/Tabla.htm?t=10822

ISO International Standard Organization (2019). The ISO Survey of Management System Standard Certifications (1993-2018) - ISO 9001 - Quality Management Systems Requirements. https://www.iso.org/the-iso-survey.html

Junta de Andalucía (2007 - 2012) Directorio de establecimientos y espacios certificados (D1, $D 2, D 3, D 4, D 5, D 6)$. Consejería de Turismo, Comercio y Deporte.

Junta de Andalucía. (2013-2016). Directorio de Establecimientos y Espacios certificados (D7, $D 8, D 9, D 10)$. Consejería de Turismo y Comercio.

Junta de Andalucía. (2017, 2018). Directorio de establecimientos y espacios certificados (D11, D12). Consejería de Turismo y Deporte.

Junta de Andalucía. (2019). Directorio de establecimientos y espacios certificados (D13). Consejería de Turismo, Regeneración, Justicia y Administración Local.

Kafel, P. y Nowicki, P. (2014). Functioning of environmental and quality management systems after resignation of management standard certification: Case study of a polish organizations. International Journal for Quality Research, 8(4), 505-516.

Kafel, P. y Simon, A. (2017). The reasons for decertification of ISO 9001: Financial aspects. Quality Innovation Prosperity, 21(3), 173-184. https://doi.org/10.12776/QIP.V21I3.1024

Marimon, F., Casadesús, M. y Heras, I. (2010). Certification intensity level of the leading nations in ISO 9000 and ISO 14000 standards. International Journal of Quality and Reliability Management, 27(9), 1002-1020. https://doi.org/10.1108/02656711011084800

Marimon, F., Heras, I. y Casadesús, M. (2009). ISO 9000 and ISO 14000 standards: A projection model for the decline phase. Total Quality Management and Business Excellence, 20(1), 1-21. https://doi.org/10.1080/14783360802614257

Mastrogiacomo, L., Carrozza, A. y Maisano, D. A. (2020). Is 'post-decline' the next phase of the diffusion of ISO 9001 certifications? New empirical evidence from European countries. Total Quality Management and Business Excellence. https://doi.org/10.1080/1478336 $\underline{3.2020 .1724508}$

Nelson, P. (1970). Information and Consumer Behavior. Journal of Political Economy. 78(2), 311-329. https://doi.org/10.1086/259630 
Nicolau, J. L. y Sellers, R. (2010). The quality of quality awards: Diminishing information asymmetries in a hotel chain. Journal of Business Research, 63(8), 832-839. https://doi. org/10.1016/j.jbusres.2009.06.009

Nwankwo, S. (2000). Quality assurance in small business organisations: Myths and realities. International Journal of Quality and Reliability Management, 17(1), 82-99. https://doi. org/10.1108/02656710010302269

Öğüt, H. y Onur Taş, B. K. (2012). The influence of internet customer reviews on the online sales and prices in hotel industry. The Service Industries Journal, 32(2), 197-214. https://doi. org/10.1080/02642069.2010.529436

Pereira-Moliner, J., Tarí, J. J., Molina-Azorín, J. F. y López-Gamero, M. D. (2016). Beneficios del certificado de calidad. Universia Business Review, 2016(50). https://doi.org/10.3232/ UBR.2016.V13.N2.04

Pertusa Ortega, E. M., Tarí Guilló, J. J., Pereira Moliner, J., Molina Azorín, J. F. y López Gamero, M. D. (2013). Certificación en calidad, resultados empresariales y estructura organizativa en el sector hotelero español. Intangible Capital, 9(1), 199-224.

Rodríguez-Arnaldo, O. y Martínez-Lorente, A. R. (2020). What determinants influence the diffusion of ISO 9001 by countries? TQM Journal. https://doi.org/10.1108/ TQM-03-2020-0055

Rubio-Andrada, L., Alonso-Almeida, M. del M. y Rodríguez-Antón, J. M. (2011). Motivations and impacts in the firm and stakeholders of quality certification: Evidence from small- and medium-sized service enterprises. Total Quality Management and Business Excellence, 22(8), 833-852. https://doi.org/10.1080/14783363.2011.593858

Rusjan, B. y Alič, M. (2010). Capitalising on ISO 9001 benefits for strategic results. International Journal of Quality and Reliability Management, 27(7), 756-778. https://doi. org/10.1108/02656711011062372

Sampaio, P., Saraiva, P. y Guimarães Rodrigues, A. (2011). ISO 9001 certification forecasting models. International Journal of Quality \& Reliability Management, 28(1), 5-26. https:// doi.org/10.1108/02656711111097526

Sánchez-Ollero, J. L., García-Pozo, A. y Marchante-Mera, A. J. (2014). Análisis de la incidencia de las certificaciones de calidad sobre la productividad de los hoteles en Andalucía. Universia Business Review, 4, 88-103.

Sansalvador, M. E. y Brotons, J. M. (2015). Valuation of the option of abandoning ISO 9001 certification: an empirical study in Spain. Total Quality Management and Business Excellence, 26(11-12), 1255-1268. https://doi.org/10.1080/14783363.2014.929231

Simon, A. y Kafel, P. (2018). Reasons for Decertification of ISO 9001. An empirical study. Innovar, 28(70), 69-80. https://doi.org/10.15446/innovar.v28n70.74449

Stigler, G. (1961). The Economics of Information. Journal of Political Economy, 69(3), 213-225. www.jstor.org/stable/1829263

Tarí, J. J., Claver-Cortés, E., Pereira-Moliner, J. y Molina-Azorín, J. F. (2009). Is it worthwhile to be a quality certified hotel? Evidence from Spain. International Journal of Quality \& Reliability Management, 26(9), 850-865. https://doi.org/10.1108/EL-01-2014-0022

Tarí, J. J., Heras-Saizarbitoria, I. y Dick, G. (2014). Internal and external drivers for quality certification in the service industry: Do they have different impacts on success? Service Business, 8(2), 337-354. https://doi.org/10.1007/s11628-013-0198-6 
Tarí-Guilló, J. J. y Pereira-Moliner, J. (2012). Calidad y rentabilidad. Análisis del certificado Q en las cadenas hoteleras. Universia Business Review, 34, 52-67.

Terlaak, A. K. y King, A. A. (2006). The effect of certification with the ISO 9000 Quality Management Standard: A signaling approach. Journal of Economic Behavior and Organization, 60(4), 579-602. https://doi.org/10.1016/i.jebo.2004.09.012

TripAdvisor (2018). TripBarometer 2017/18 Global Report. https://www.tripadvisor.com/ TripAdvisorlnsights/wp-content/uploads/2018/10/TripBarometer-2017-2018.pdf

Tsao, W. C., Hsieh, M. T., Shih, L. W. y Lin, T. M. Y. (2015). Compliance with eWOM: The influence of hotel reviews on booking intention from the perspective of consumer conformity. International Journal of Hospitality Management, 46, 99-111. https://doi. org/10.1016/i.ijhm.2015.01.008

Zhao, X. (Roy), Wang, L., Guo, X. y Law, R. (2015). The influence of online reviews to online hotel booking intentions. International Journal of Contemporary Hospitality Management, 27(6), 1343-1364. https://doi.org/10.1108/IJCHM-12-2013-0542

Zimon, D. y Dellana, S. (2019). A longitudinal exploratory study of ISO 9001 certification abandonment in small- and medium-sized enterprises. International Journal of Quality and Reliability Management, 37(1), 53-67. https://doi.org/10.1108/IJQRM-10-2018-0284

\section{AUTORÍA}

Inmaculada Rabadán-Martín Concepción original del trabajo; Análisis, adquisición e interpretación de los datos; Redacción y revisión crítica del contenido y; Aprobación final de la versión a publicar. Francisco Aguado-Correa Análisis, adquisición e interpretación de los datos; Redacción y revisión crítica del contenido y; Aprobación final de la versión a publicar. Nuria Padilla-Garrido Análisis, adquisición e interpretación de los datos; Redacción y revisión crítica del contenido y; Aprobación final de la versión a publicar

\section{AGRADECIMIENTOS}

No se ha contado con fuentes de financiación 